University of Nebraska - Lincoln

DigitalCommons@University of Nebraska - Lincoln

8-1-1989

\title{
The Response of the Alanine Detector After Charged-Particle and Neutron Irradiations
}

\author{
M. P. R. Waligorski \\ Institute of Nuclear Physics, Radzikowskiego 152, PL 31-342 Krakow, Poland \\ G. Danialy \\ University of Nebraska-Lincoln \\ Robert Katz \\ University of Nebraska-Lincoln, rkatz2@unl.edu
}

Follow this and additional works at: https://digitalcommons.unl.edu/physicskatz

Part of the Physics Commons

Waligorski, M. P. R.; Danialy, G.; and Katz, Robert, "The Response of the Alanine Detector After ChargedParticle and Neutron Irradiations" (1989). Robert Katz Publications. 98.

https://digitalcommons.unl.edu/physicskatz/98

This Article is brought to you for free and open access by the Research Papers in Physics and Astronomy at DigitalCommons@University of Nebraska - Lincoln. It has been accepted for inclusion in Robert Katz Publications by an authorized administrator of DigitalCommons@University of Nebraska - Lincoln. 


\title{
The Response of the Alanine Detector After Charged-Particle and Neutron Irradiations
}

\author{
M. P. R. Waligórski, ${ }_{1}^{1}$ G. Danialy, ${ }^{2}$ Kim Sun Loh, ${ }^{2}$ and R. Katz ${ }^{2}$ \\ ${ }^{1}$ Institute of Nuclear Physics, Radzikowskiego 152, PL 31-342 Krakow, Poland \\ 2 University of Nebraska-Lincoln, Lincoln, NE 68588, U.S.A.
}

\begin{abstract}
Radiosensitivity parameters of track structure theory, representing alanine as a one-hit detector, have been fitted for this free-radical amino-acid system on the basis of the available experimental data on the relative effectiveness of alanine after charged particle and neutron irradiations. The experimental data set can be reproduced by theoretical calculations, roughly to within experimental accuracy. A charged-particle "equivalent radiation" is introduced which can mimic the response of alanine to neutron irradiations. Implications of the results of model calculations for alanine on the shape of the radial distribution of $\delta$-ray dose postulated by track theory, are discussed.
\end{abstract}

\section{Introduction}

Alanine dosimetry has now reached a stage of development which could make it an attractive alternative to the ferrous sulphate (Fricke) system with respect to accuracy, repeatability, and useful dose range. The chemical composition of this amino-acid ( $\mathrm{L}-\alpha$-alanine: $\mathrm{CH}_{3}$. $\left.\mathrm{CH}\left(\mathrm{NH}_{2}\right) \cdot \mathrm{COOH}\right)$ is quite close to that of tissue, which makes alanine very suitable for neutron dosimetry. Among the radical species generated in alanine by ionizing radiation, the stable radical $\mathrm{CH}_{3}-{ }^{\cdot} \mathrm{CH}-\mathrm{COOH}$, which is predominant at room temperature, gives a prominent pattern when measured using an electron spin resonance (ESR) spectrometer. The concentration of this free radical measured by ESR-spectrometry represents the dose absorbed in the alanine dosimeter.

In order for a dosimetric system to be applicable in practice, an accompanying model is required which can analyze and predict the response of this system to radiations of all qualities. Track structure theory (Katz et al., 1972a,b; Katz, 1978), which relates the signal of a detector after doses of heavy charged particles with its signal after doses of $\gamma$-rays or energetic electrons, is able to fulfill these requirements.

Alanine was first used as a solid-state dosimeter 25 years ago. Bradshaw et al. (1962) developed this system for measurements of $x, \gamma, \beta$, and proton doses. Rotblat and Simmons (1963) found that a simple exponential formula describes the response of alanine after $\gamma$-ray doses. Snipes and Horan (1967) studied the free-radical kinetics in $\gamma$-ray irradiated alanine and concluded that radical saturation in alanine at ${ }^{60} \mathrm{Co}$ doses above $10^{5} \mathrm{~Gy}$ is due to a process of radical destruction by radiation. Thus, the exponential formula, or the identical "onehit" formula of Katz's track strcuture theory (Katz et al., 1972a), used to represent alanine as a one-hit detector, can be justified by the kinetics of radical formation or by the one-or-more-hit statistics of "sensitive-site inactivation" (Dertinger and Jung, 1970).

The relative effectiveness* of alanine to different radiation qualities, including photons, $\gamma$-rays, energetic heavy ions (ranging from ${ }^{10} \mathrm{~B}$ to ${ }^{40} \mathrm{Ar}$, of energy 10.4 $\mathrm{MeV} / \mathrm{a} . \mathrm{m} . u$.), lighter ions of lower energies, and neutrons, has been measured by several authors. (Ebert et al., 1965; Henriksen, 1966; Mueller et al., 1964; Simmons and Bewley, 1976; Bermann, 1978; Katz and Bermann, 1976; Deffner and Regulla, 1980; Regulla and Deffner, 1982; Waligórski et al., 1981; Hansen and Olsen, 1985; Hansen, 1984; Hansen et al., 1987; Katsumura et al., 1986; Waligórski et al., 1987a).

A problem of some importance in practical applications, namely the production of mechanically stable detectors containing alanine powder in a form suitable for ESR measurements, has been solved. One technique, developed by Bermann (1978), Katz and Bermann (1976), and by Deffner and Regulla $(1980,1982)$ is to mix alanine powder with paraffin; another, introduced by Waligórski

* Effectiveness relative to ${ }^{60} \mathrm{Co}$ (RE), i.e. the ratio of signals after equal doses of a given radiation quality and of ${ }^{60} \mathrm{Co} \gamma$ rays. In the linear range of the signal-dose dependence, RE is equal to RBE, i.e. to the ratio of ${ }^{60} \mathrm{Co} \gamma$-ray dose and dose of radiation of a given quality required to obtain the same signal in the detector. 
et al. (1981) and further improved by Hansen and Olsen (1987), is to press alanine power with a suitable cellulose binder to form stable tablets.

Track structure calculations of the response* of alanine after irradiations with protons, a-particles and fast neutrons were made by Katz and Herman (1976) using the model described by Butts and Katz (1967). Since then several improvements in the formulation of the radial distribution of dose, a central element of the theory, were proposed by Zhang et al. (1985) and by Waligórski et al. (1987b). This last formulation led to a better theoretical description of the inactivation of dry enzymes and viruses (Waligórski et al., 1987c) and of the Fricke dosimeter (Katz et al., 1986) after heavy-ion bombardments, both systems being described as one-hit detectors.

Hansen and Olsen (1984) have chosen a different way of calculating the activation cross-section and used another algorithm describing the radial distribution of dose of track structure theory. Their theoretical calculations fit their experimental results (Hansen and Olsen, 1985; Hansen, 1984; Hansen et al., 1987).

The aims of our work were twofold. By gathering the available data on the response of the alanine detector to charged-particle and neutron irradiations, we were able to check their consistency and acquire a basis for fitting radiosensitivity parameters of alanine.

In our calculations we used the recent formulation of the radial distribution of dose (Waligórski et al., 1987b). We therefore expected to verify the correctness of this formulation and gain indirect information on the shape of the radial distribution of dose around the path of a heavy ion.

\section{Modeling the Response of a One-hit Detector}

Track structure theory (Katz et al., 1972a,b; Katz, 1978) relates the response of a detector after doses of "test" radiation (usually ${ }^{60} \mathrm{Co} \gamma$-rays) to its response after doses of heavy charged particles, specified by their charge, velocity, and fluence. The response of a one-or-morehit detector after a dose of test radiation is assumed to obey the cumulative Poisson distribution (Dertinger and Jung, 1970). In the case of alanine, this translates to the formula of Rotblat and Simmons (1962):

$$
P(D)=S(D) / S_{0}=1-\exp \left(-D / E_{0}\right)
$$

where $P(D)$ is the probability that "action" after a dose, $D$, occurs, $S(D)$ and $S_{0}$ are the numbers of free radicals (per unit weight of alanine sample) after a dose $D$ and at saturation respectively, and $E_{0}$ is the dose of test radiation at which there is an average of one "hit" per target (Dertinger and Jung, 1970).

\footnotetext{
* Signal vs dose dependence.
}

The sensitive elements or targets in the detector are assumed to be spheres [or chunky cylinders (Butts and Katz, 1967)] of radius, $a_{0}$, the "(in)activation" of which is described by the average dose over their volume. Track structure theory assumes that (in)activation of these targets is due to "hits" (Dertinger and Jung, 1970) by secondary electrons irrespectively of whether they originate from $\gamma$-rays or from $\delta$-rays surrounding the path of an energetic heavy ion traversing the detector medium. In the latter case knowledge of the radial distribution of average dose due to $\delta$-rays, $D(r)$, in concentric cylinders around the ion's path is necessary. The presently used form (Waligórski et al., 1987), described in the Appendix, is:

$$
D(r)=D_{1}(r)[1+K(r)]
$$

where $D_{1}(r)$ is the form developed by Zhang et al. (1985), which, compared to the original formula of Butts and Katz (1967), exploits a power-law range-energy relationship for electrons and a correction in the Rutherford cross-section for $\delta$-ray production from atoms having ionization potential $I=10 \mathrm{eV}$. Normal ejection of $\delta$-rays up to a maximum, kinematically constrained, range, $\tau$, (see Appendix) is assumed.

As $D(r)$ falls of rapidly, approximately inversely with the square of the radial distance from the ion's path, the average dose over the volume of the sensitive element, whose centre is placed at a distance $t$ from the ion's path, $E(t)$, has to be calculated. The radial distribution of activation probability $P(t)$ can then be introduced:

$$
P(t)=P(E(t))=P\left(1-\exp \left(-E(t) / E_{0}\right)\right),
$$

which involves $D(r)$ and $a_{0}$ and represents the radially-dependent probability that a sensitive site whose center lies at a distance $t$ from the ion's path will be (in)activated. The single-particle (in)activation cross-section a is now calculated by integrating $P(t)$ over all distances:

$$
\sigma=\int_{0}^{t+a_{0}} 2 \pi t P(t) \mathrm{d} t
$$

where, in practice, the upper range of integration is limited to the maximum radial penetration of $\delta$-rays, $\tau$.

After a fluence, $F$, particles/ $\mathrm{cm} 2$, or ion dose (in water) $D_{\mathrm{i}}=F \times L$ ( $L$ is the stopping power of the ion, or its LET), the signal observed in a thin specimen (track-segment irradiation) is:

$$
\begin{aligned}
S\left(D_{\mathrm{i}}\right) & =S_{0}[1-\exp (-\sigma F)] \\
& =S_{0}\left[1-\exp \left(-\sigma D_{\mathrm{i}} / L\right)\right] .
\end{aligned}
$$

The relative detector effectiveness (RE) is defined as the ratio of detector signals after equal doses of ion and test $\left({ }^{60} \mathrm{Co}\right)$ radiations, i.e.:

$$
\mathrm{RE}=S\left(D_{\mathrm{i}}\right) / S\left(D_{\mathrm{g}}\right) ; D_{\mathrm{i}}=D_{\mathrm{g}} .
$$

After expanding equations (1) and (6) in the linear region and performing simple arithmetic, we arrive at the 
following description of relative effectiveness in track segment irradiation:

$$
\operatorname{RE}(\text { calculated })=\sigma E_{0} / L \text {. }
$$

This value can be compared with values of relative effectiveness measured experimentally, according to equation (5), by irradiating thin samples with heavy ions, i.e. samples whose thickness is much smaller than the ion's range in the detector.

A thick sample is one whose thickness exceeds the range of the ion in the detector.

To calculate the response of a detector to stopping ions of initial kinetic energy $T_{\mathrm{i}}$ and of range $R$, we integrate the response over the pathlength. We have therefore to replace $\sigma$ and $L$ in equation (7) by their average values:

$$
\sigma_{\mathrm{AVE}} R=\int_{0}^{R} \sigma \mathrm{d} r=\int_{T_{\mathrm{i}}}^{0}(\sigma / L) \mathrm{d} T
$$

and:

$$
L_{\mathrm{AVE}}=T_{\mathrm{i}} / R \text {. }
$$

The effect of irradiating the detector with a beam of fast neutrons is calculated by adding the contributions from all the charged secondaries stopping in the medium (Katz et al., 1972a,b). Let us denote the number of neutron interactions per cubic centimeter of detector volume by $Y$, and the absorbed dose from neutrons by $D_{\mathrm{n}} \cdot R_{\mathrm{Zi}}$ represents the range (in $\mathrm{cm}$ ) of an ion of atomic number $Z$ and initial kinetic energy $T_{\mathrm{i}}$, while $\mathrm{d} N_{\mathrm{Zi}} / \mathrm{d} T_{\mathrm{i}}$ represents the number of secondary charged particles of atomic number $Z$ and initial kinetic energy $T_{i}$ per unit initial kinetic energy interval per neutron interaction per cubic centimeter of detector. Then:

$$
D_{\mathrm{n}}=Y\left[\sum_{Z} \sum_{T_{\mathrm{i}}}\left(\Delta T_{\mathrm{i}}\right)\left(\mathrm{d} N_{Z_{\mathrm{i}}} / \mathrm{d} T_{\mathrm{i}}\right) T_{\mathrm{i}}\right]
$$

and

$$
\begin{aligned}
P\left(D_{\mathrm{n}}\right)=1-\exp \{ & -Y\left[\sum_{Z} \sum_{T_{\mathrm{i}}}\left(\Delta T_{\mathrm{i}}\right)\right. \\
& \left.\left.\times\left(\mathrm{d} N_{\mathrm{Zi}_{\mathrm{i}}} / \mathrm{d} T_{\mathrm{i}}\right) \sigma_{\mathrm{AVE}, \mathrm{Zi}} R_{\mathrm{Zi}}\right]\right\} .
\end{aligned}
$$

The detector signal after a neutron dose $D_{\mathrm{n}}$ is then $S\left(D_{\mathrm{n}}\right)$ $=S_{0} P\left(D_{\mathrm{n}}\right)$, and the relative effectiveness of the detector after a neutron dose can be calculated by replacing $D_{\mathrm{i}}$ by $D_{\mathrm{n}}$ in equation (6) and compared with experiment.

For details on the model and on the neutron calculation, the review papers of Katz et al. (1972a,b; 1978) should be consulted. The currently used set of formulae to describe the radial distribution of dose $D(r)$ has been elaborated elsewhere (Waligórski et al., 1987b) and is given in the Appendix. For an overview of the track structure model developed by Katz, see Waligórski (1987).

\section{Results}

According to the requirements of the model calculation, experimental data were grouped into the three categories: charged particles stopping in the detector (Table 1 ), track segment irradiations (Table 2), and neutron irradiations (Table 3). Except for data of Hansen and Olsen (1985), Hansen et al. (1987), and of Hermann (1978), which have been reported in a form directly applicable for comparison with model calculations, values of ex-

Table 1. Measured and calculated values of relative effectiveness (RE) of alanine irradiated by charged particles stopping in the detector.

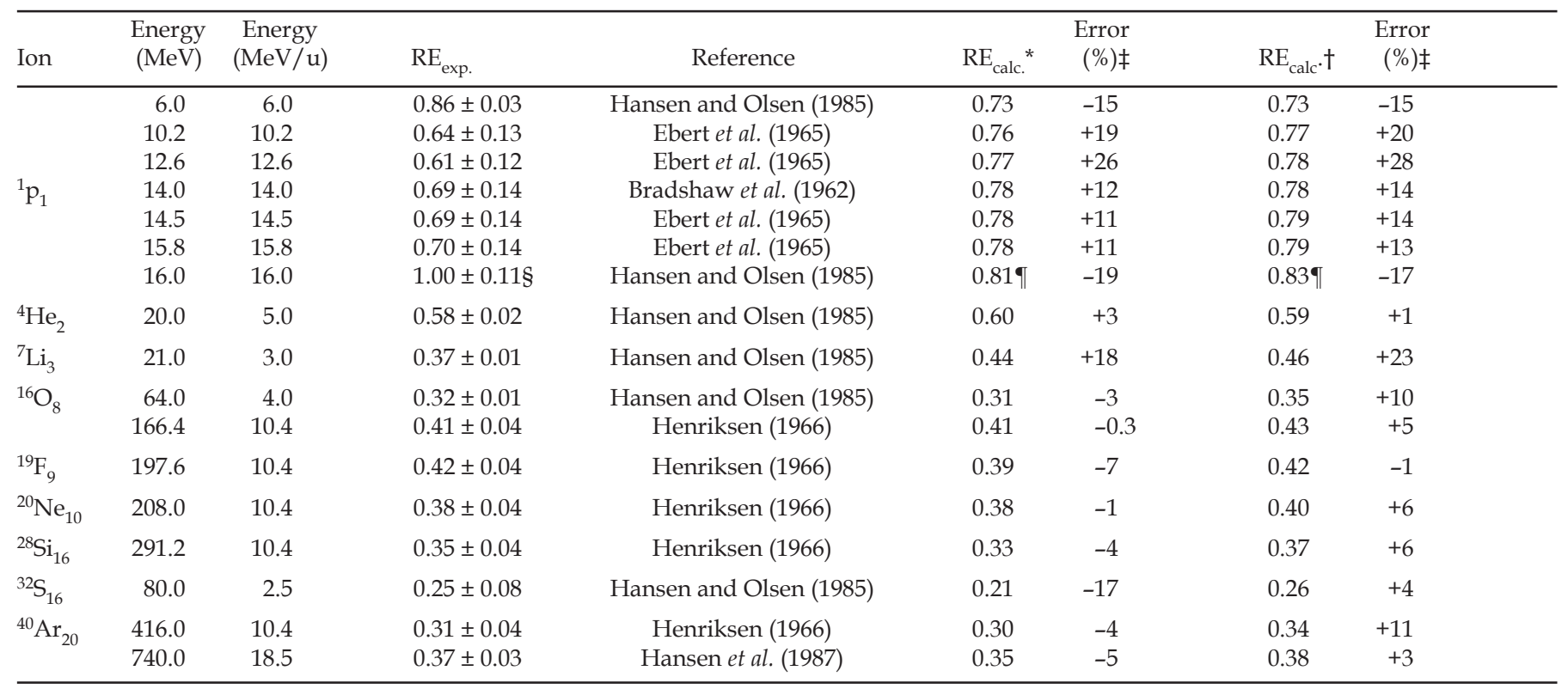

${ }^{*} E_{0}=1.05 \times 10^{5} \mathrm{~Gy}, a_{0}=2.0 \mathrm{~nm}, \tau \rightarrow \tau / 2$ (see text and Appendix).

$\dagger E_{0}=7.5 \times 10^{4} \mathrm{~Gy}, a_{0}=0.5 \mathrm{~nm}$.

$\ddagger$ Error $=100 \% \times\left(\mathrm{RE}_{\text {calc }}-\mathrm{RE}_{\text {exp }}\right) / \mathrm{RE}_{\text {exp }}$.

$\S$ Partially stopping particle of full range $0.29 \mathrm{~g} / \mathrm{cm}^{2}$ in an analine detector of thickness $0.24 \mathrm{~g} / \mathrm{cm}^{2}$ (density of alanine $\rho=1.21 \mathrm{~g} / \mathrm{cm}^{3}$, thickness of alanine detector $2.0 \mathrm{~mm}$ ).

- Calculated assuming initial proton energy $T_{\mathrm{i}}=16 \mathrm{MeV}$, final proton energy $T_{\mathrm{f}}=8.3 \mathrm{MeV}$, from equations (7) and (8). 
Table 2. Measured and calculated values of relative effectiveness (RE) of alanine irradiated by charged particles in track segment mode

\begin{tabular}{|c|c|c|c|c|c|c|c|c|}
\hline Ion & $\begin{array}{l}\text { Energy } \\
(\mathrm{MeV} / \mathrm{u})\end{array}$ & $R E_{\text {exp }}$ & Reference & $\begin{array}{c}\text { Speed } \\
(\beta=v / c)\end{array}$ & $\mathrm{RE}_{\text {calc. }}{ }^{*}$ & $\begin{array}{l}\text { Error } \\
(\%) \ddagger\end{array}$ & $\operatorname{RE}_{\text {calc. }} \dagger^{\dagger}$ & $\begin{array}{r}\text { Error } \\
(\%) \ddagger\end{array}$ \\
\hline${ }^{1} \mathrm{p}_{1}$ & 160 & 1.0 & Bermann (1978) & 0.521 & 0.91 & -9 & 0.93 & -7 \\
\hline${ }^{4} \mathrm{He}_{2}$ & 162.5 & 1.0 & Bermann (1978) & 0.524 & 0.91 & -9 & 0.93 & -7 \\
\hline
\end{tabular}

${ }^{*} E_{0}=1.05 \times 10^{5} \mathrm{~Gy}, a_{0}=2.0 \mathrm{~nm}, \tau \rightarrow \tau / 2$ (see text and Appendix).

$\dagger E_{0}=7.5 \times 10^{4} \mathrm{~Gy}, a_{0}=0.5 \mathrm{~nm}$.

$\ddagger$ Error $=100 \% \times\left(R E_{\text {calc }}-R E_{\text {exp }}\right) / R E_{\text {exp }}$.

perimental data points for stopping particles had to be extracted from results published by other authors.

To evaluate data concerning irradiation of alanine powder by protons stopping in the detector reported by Ebert et al. (1965), average values of relative effectiveness, in arbitrary units, were read from Figure 7 of their paper by dividing the respective ordinate values (ESR response/g) by the values on the abscissa (proton fluence) multiplied by initial proton energy. The value of relative effectiveness for $14 \mathrm{MeV}$ protons stopping in alanine powder evaluated from Figures 3 and 5 of the paper by Bradshaw et al. (1962), $\mathrm{RE}=0.69$, was then used to convert these values into data points listed in Table 1.

Henriksen (1966) reports values of radical yield (in relative units) for alanine powder samples of thickness $20-50 \mathrm{mg} / \mathrm{cm}^{2}$ irradiated by a range of heavy ion species of energies $10.4 \mathrm{MeV} /$ a.m.u. without specifying which of these results concern particles fully stopped in the detector. We have therefore calculated the ranges and average values of LET for all these bombardments from equation (9), and compared them with LET values plotted in Figure 2 of Henriksen's paper. We accepted all but the three lightest ions $(\mathrm{He}, \mathrm{Li}$, and $\mathrm{B})$ as particles stopping in the detector and listed these data in Table 1.
We have arbitrarily assumed the relative accuracy of the obtained data points to be $20 \%$ for the proton data and $10 \%$ for the remaining data points, except when reported (Hansen and Olsen, 1985; Hansen et al., 1987), and we list the respective absolute errors with each extracted data point in Table 1. The values of relative effectiveness extracted from the paper of Bradshaw et al. (1962) for 5 and $7.7 \mathrm{MeV}$ protons $(\mathrm{RE}=0.23$, and $\mathrm{RE}=$ 0.49 , respectively) were rejected as being inconsistent with adjacent data reported in their paper.

The experimental values of relative effectiveness for track segment irradiations of alanine (Table 2) are based on the irradiations of alanine with $160 \mathrm{MeV}$ protons from the Harvard cyclotron and with $650 \mathrm{MeV} \alpha$ particles from the cyclotron at Saclay, reported by Bermann (1978). The energy value of 58.6 MeV/a.m.u. was calculated from the lowest range of the $\alpha$ particle $(3 \mathrm{~cm}$ upstream of the $650 \mathrm{MeV}$ beam's residual range), at which the measured value of relative effectiveness of alanine was still 1.0.

The experimental data for alanine irradiated by neutrons (Table 3) was gathered from the papers of Bermann (1978), Waligórski et al. (1981, 1987a), and Katsumura et al. (1986).

Table 3. Measured and calculated values of relative effectiveness of alanine irradiated by neutrons

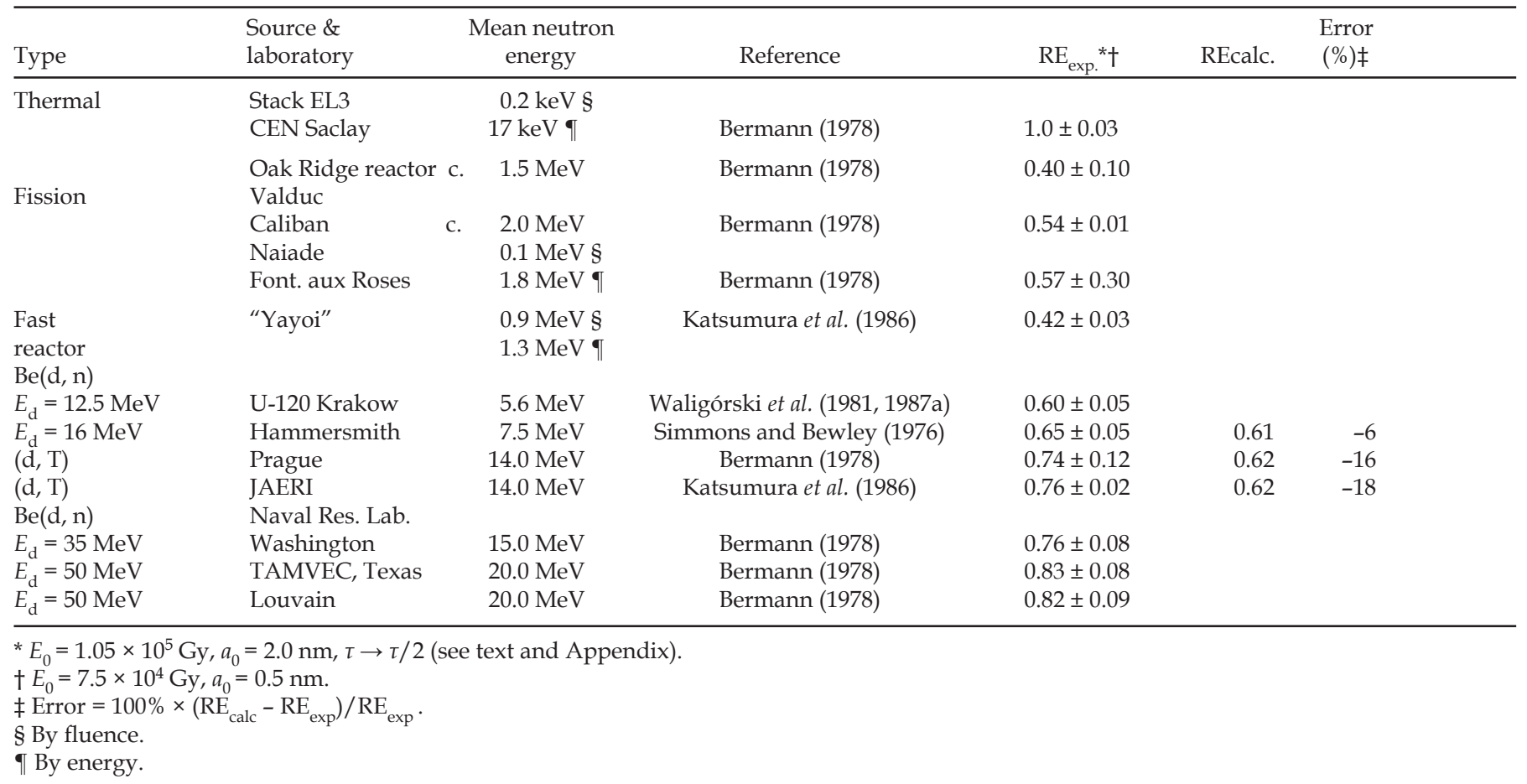




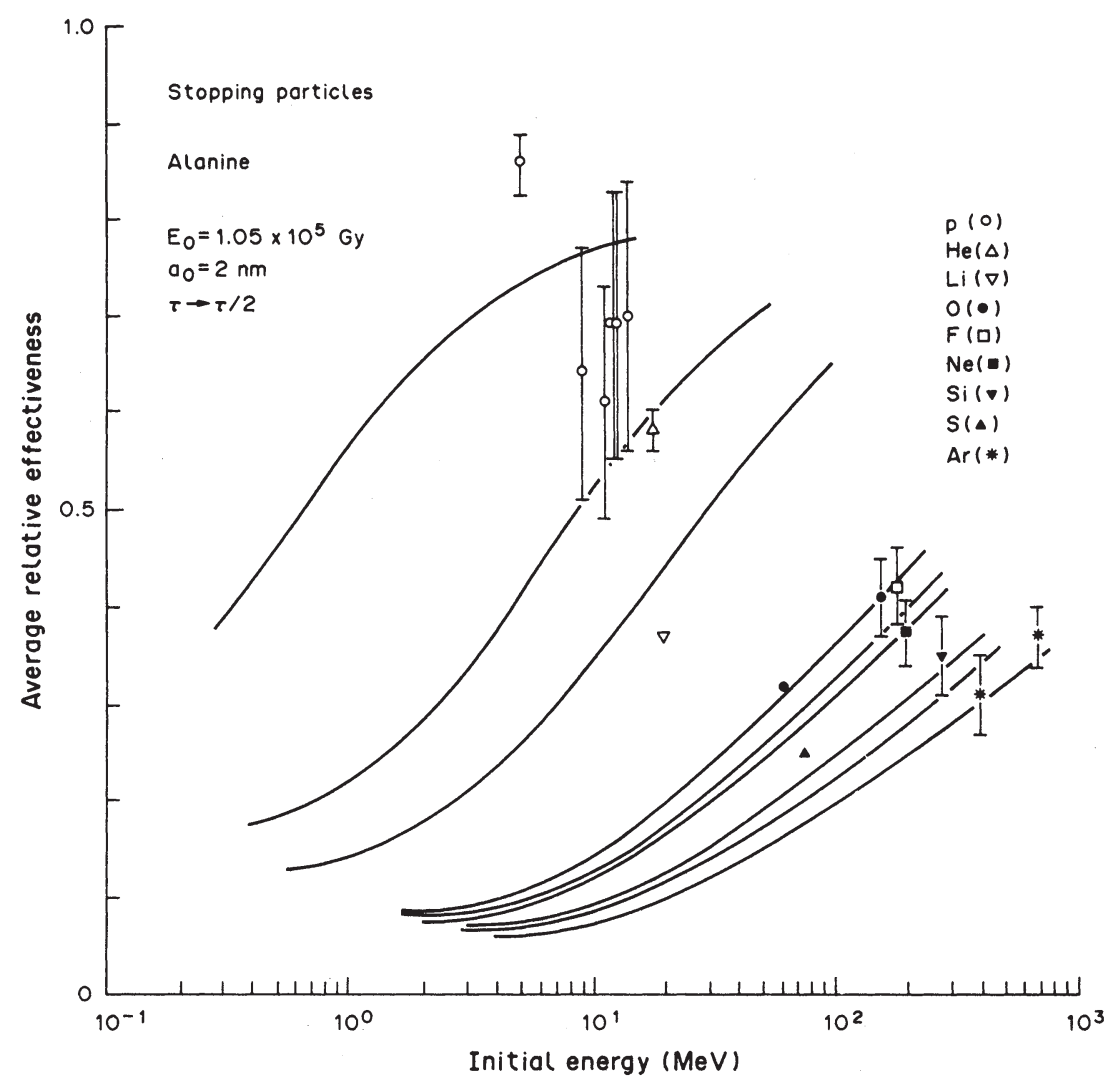

Figure 1. Measured and calculated average relative effectiveness of alanine vs. initial energy (MeV) of charged particles stopping in the detector. For sources of experimental data, see Table 1 . The radiosensitivity parameters of alanine used in the calculations are: $c=1, E_{0}=1.05 \times 10^{5} \mathrm{~Gy}, a_{0}=2 \mathrm{~nm}$. In this calculation, the maximum radial penetration $\tau$ in the dose distribution formula [equation (2) and equation (A.10)] has been reduced by a factor $2(\tau \rightarrow \tau / 2)$.

As mentioned earlier, in track theory calculations the $c$ hit detector is specified by three radiosensitivity parameters: "hittedness" $c$ (for alanine, $c=1$ ), characteristic $\gamma$ ray dose $E_{0}$, and radius of sensitive site, $a_{0}$.

The value of $E_{0}$ has been assessed by several authors who studied the response of alanine after $\gamma$-ray and electron irradiation. Values of $E_{0}=1.17 \times 10^{5} \mathrm{~Gy}, 1.10 \times 10^{5}$ Gy, $7.5 \times 10^{4} \mathrm{~Gy}$, and $1.05 \times 10^{5}$ Gy were found by Snipes and Horan (1967), Hermann (1978), Waligórski et al. (1981), Hansen and Olsen (1985) and Hansen (1984), respectively. This last value is probably the most accurate.

Using a fixed value of $E_{0}=1.05 \times 10^{5}$ Gy and varying the value of $a_{0}$, we were unable to find a satisfactory fit to the experimental data set listed in Tables 1 and 2. We therefore modified the shape of the radial distribution of dose [equation (2)] by limiting its maximum radial penetration ( $\tau$, see Appendix) to one-half of the hithertoused value and we repeated our search for the best fitting value of $a_{0}$. The results of our calculations where the alanine detector is represented by $c=1, E_{0}=1.05 \times$ $10^{5} \mathrm{~Gy}, a_{0}=2 \mathrm{~nm}$ and $(\tau \rightarrow \tau / 2)$ are given in Table 1, Figure 1 , and Table 2.

We have also searched for alanine radiosensitivity parameters by optimizing both values of $E_{0}$ and $a_{0}$, without modifying the value of $\tau$, i.e. using the original shape of the radial distribution of dose $D(r)$ [equation (2)] in our calculations. The best fitting values are then $E_{0}=7.5 \times$ $10^{4} \mathrm{~Gy}$ and $a_{0}=0.5 \mathrm{~nm}$. Results of this version of our calculations are also given in Tables 1 and 2.

Model calculations of the response of alanine after neutron irradiations rely on the availability of energy spectra of secondary charged particles generated in alanine by the neutron beams. Spectra of secondary particles generated in tissue by the fast neutron beam of the MRC Hammersmith cyclotron have been kindly provided to us (Dr. John A. Dennis, private communication, 1971). Bach and Caswell (1968) and Caswell and Coyne (1972) have calculated secondary charged particle spectra generated in tissue by monoenergetic neutrons in the range $60 \mathrm{keV}$ to $2 \mathrm{MeV}$ and for $14 \mathrm{MeV}$ neutrons, and also kindly provided us with their results (Dr. Randall S. Caswell, private communication, 1972). The neutron programs, originally developed by Ms. Rose Anne RothKrauter for the IBM mainframe (Rose Ann Krauter, “The Sigma Integration," UN-L, Lincoln, NE 1977, unpublished) have been adapted to run on the IBM PC/XT microcomputer.

Neutron calculations were made using both representations of the alanine detector and results were found to be almost identical (within 1\%). These results are given 


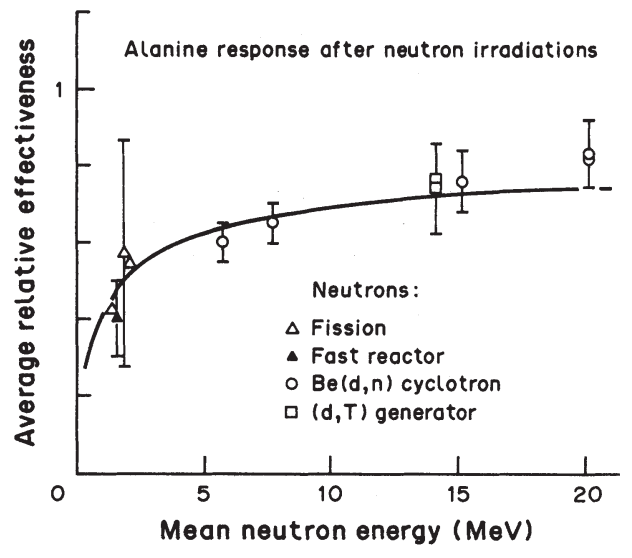

Figure 2. Measured average relative effectiveness of alanine after neutron irradiation vs. neutron energy $(\mathrm{MeV})$. The line is the average relative effectiveness calculated for a "particle" of charge $Z=1.5$ and mass $A=3$ a.m.u. stopping in the detector, vs. the "particle's" initial energy (MeV/a.m.u.). The parameters of alanine and the radial dose distribution used in this calculation are those given in caption to Figure 1.

in Table 3 and in Figure 2. The results of calculations of the response of alanine to beams of monoenergetic neutrons of energies in the range from $60 \mathrm{keV}$ to $2 \mathrm{MeV}$ are given in Figure 3.

We have made an attempt at finding a radiation "equivalent" to neutron beams, i.e. a particle of initial energy T, (MeV/a.m.u.) stopping in the detector which would yield relative effectiveness of alanine similar to that measured or calculated for a neutron beam of the same mean energy $E_{\mathrm{n}}(\mathrm{MeV})$. We found that a "particle" of charge $Z=1.5$ and mass 3.0 a.m.u. fulfilled these requirements. Except for the neutron resonance energy regions, one could use a simple stopping particle calculation using this "equivalent radiation" to estimate the response of alanine from a known energy spectrum of a neutron beam even if secondary charged particle spectra were unavailable for making a calculation according

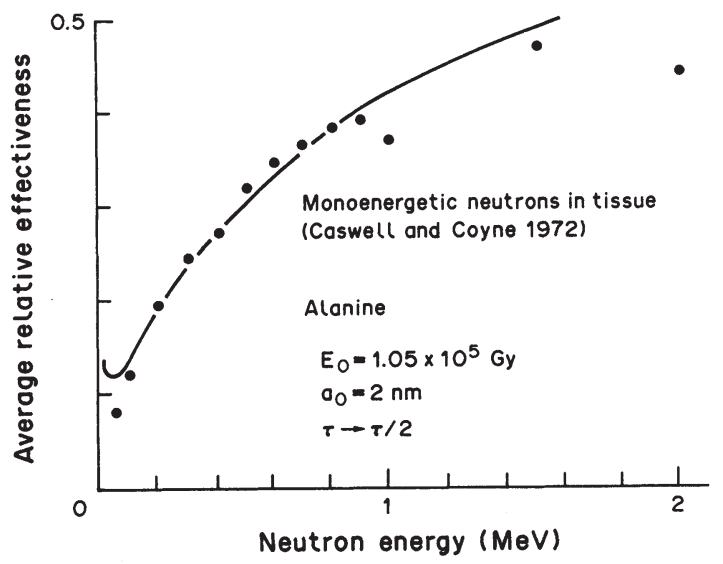

Figure 3. Calculated average relative effectiveness of alanine after irradiation by monoenergetic neutrons of energies ranging from $60 \mathrm{keV}$ to $2 \mathrm{MeV}$. The line is the average relative effectiveness calculated for the "particle" of Figure 2 using alanine parameters and the radial dose distribution given in the caption to Figure 1. to equations (10) and (11). Results of these "equivalent radiation calculations $\left(\mathrm{c}=1, E_{0}=1.05 \times 10^{5} \mathrm{~Gy}, a_{0}=2\right.$ $\mathrm{nm}, \tau \rightarrow \tau / 2$ ) are shown in Figures 2 and 3.

\section{Discussion}

Our theoretical calculations reproduce the experimental data set with an overall accuracy of about $15 \%$, except for the proton exposures, where agreement is less satisfactory, albeit, over a rather inconsistent data set (see Table 1). In general, the more energetic and heavier the particle, the better is the agreement between our model and the experiment. The calculation appears to underestimate the measured response of alanine to protons and $\alpha$-particles, for stopping (Table 1) and track segment (Table 2) irradiations of alanine, by $10-15 \%$. Both versions of our calculations appear to fit the data in a similar fashion. It is more difficult to judge the ability of our calculations to reproduce the neutron response of alanine. Where secondary spectra and measured response were available (MRC cyclotron and $14 \mathrm{MeV}$ generator beams), the calculated results underestimate those measured, by $6 \%$ and ca. $20 \%$ respectively (Table 3 ). The "equivalent radiation" calculation appears to underestimate the response of alanine to $20 \mathrm{MeV}$ cyclotron-produced neutron beams by ca. $15 \%$.

The importance of choosing suitable parameters for displaying experimental data and interpreting them theoretically is illustrated in Figure 4. Here, the experimental data set and the results of our stopping particle calculations of Table 1 and Figure 1 are presented as a plot of average relative effectiveness vs. average LET [equation (9)]. To draw any conclusions about the general dependence of relative effectiveness on LET by interpolating between the data points plotted in this figure (Henriksen, 1966; Simmons, 1987) is not only inappropriate but is highly misleading. The steep dependence of average relative effectiveness on average LET for any one ion species is demonstrated by the experimental results of Henriksen (1966) and of Hansen and Olsen for oxygen (1985) and argon (Henriksen, 1966; Hansen et al., 1987) ions.

The implications of our results with respect to the assumed shape of the radial distribution of dose $D(r)$ [equation (2)] appear to be quite interesting.

Our modification of the formula developed by Zhang et al. (1985), whereby the term $K(r)$ added in equation (2) (see also Appendix), is designed to reproduce the value of the ion's unrestricted stopping power, LET (Waligórski et al., 1987b):

$$
\int_{0}^{\tau} 2 \pi r D(\beta, Z ; r) \mathrm{d} r=\operatorname{LET}(\beta, Z)
$$

The effect of this term is seen in Figure 5, where calculations of average relative efficiency for stopping particles using Zhang and Katz's formula [equation (A.4)] 


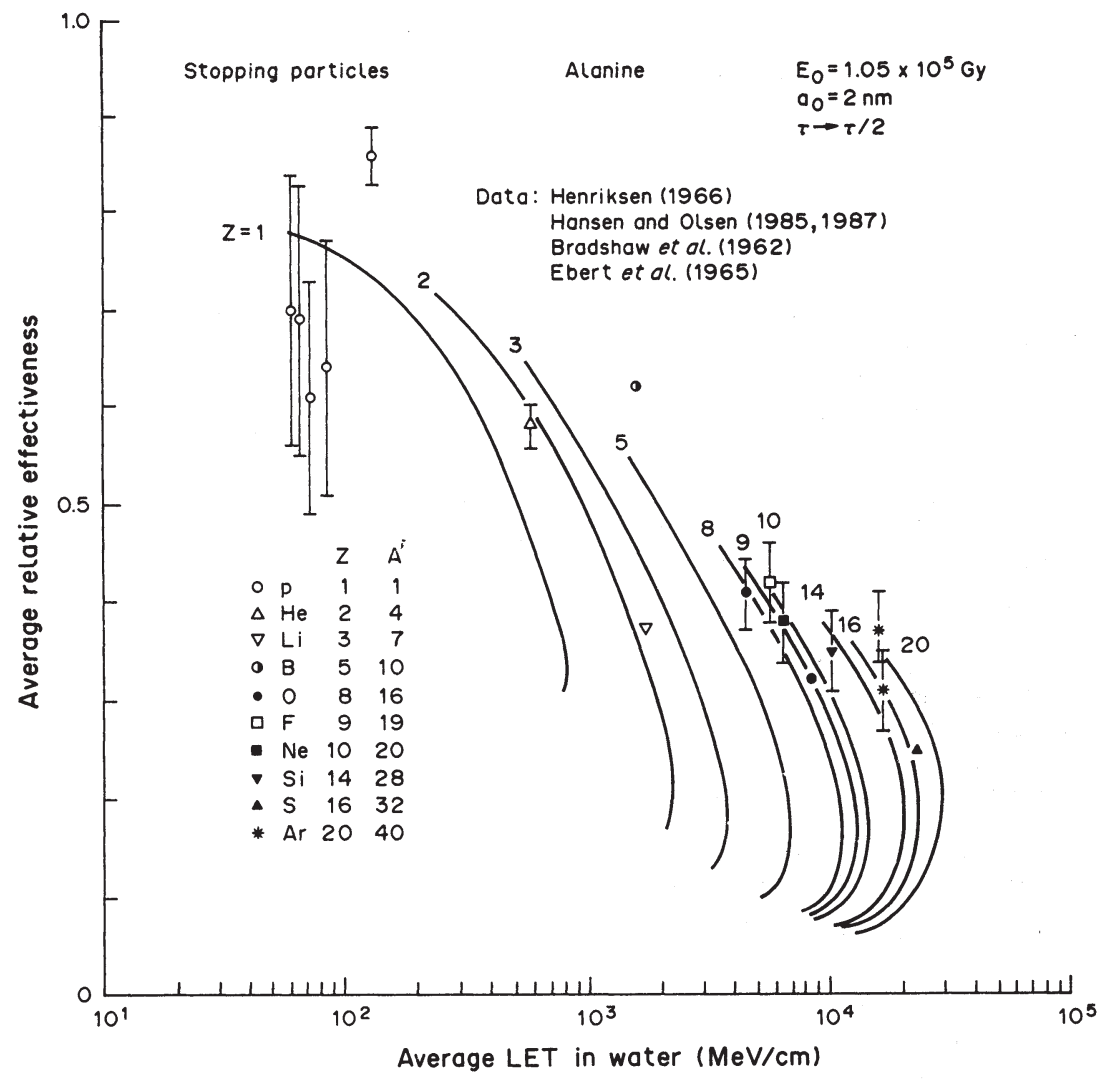

Figure 4. Measured and calculated average relative effectiveness of alanine vs. average LET, in water, of charged particles stopping in the detector. The data displayed are those of Figure 1 and Table 1, including a data point and calculation for a $10.4 \mathrm{MeV} /$ a.m.u. B ion(Henriksen, 1966). The alanine parameters and the radial dose distribution used in these calculations are those given in the caption to Figure 1.

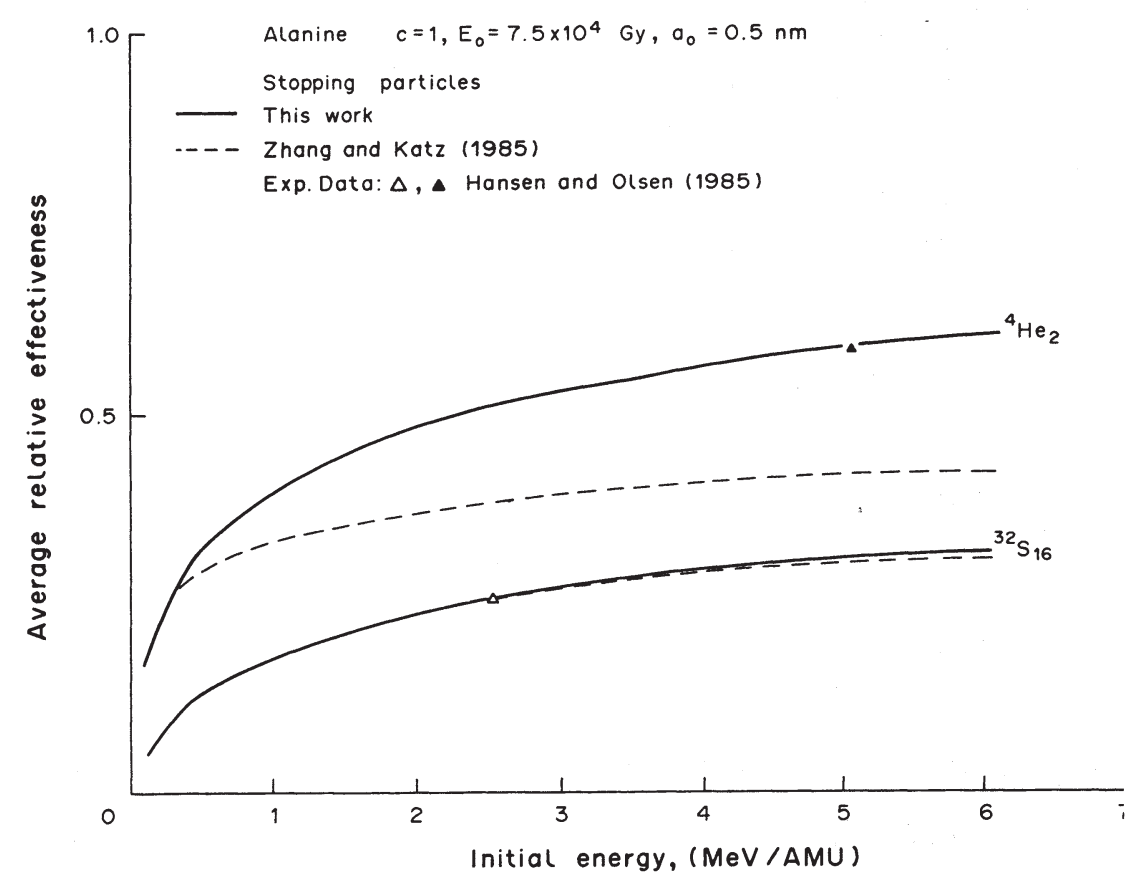

Figure 5. Calculated average relative effectiveness of alanine vs. initial energy (MeV/a.m.u.) of He and $\mathrm{S}$ ions stopping in the detector. The radiosensitivity parameters of alanine used in this calculation are: $c=1, E_{0}=7.5 \times 10^{4} \mathrm{~Gy}, a_{0}=0.5 \mathrm{~nm}$. The full line is a result of a calculation using the radial distribution of dose given by equation (2); the broken line of that using the radial distribution without the term $K(r)$ in equation (2) (Zhang et al., 1985). 


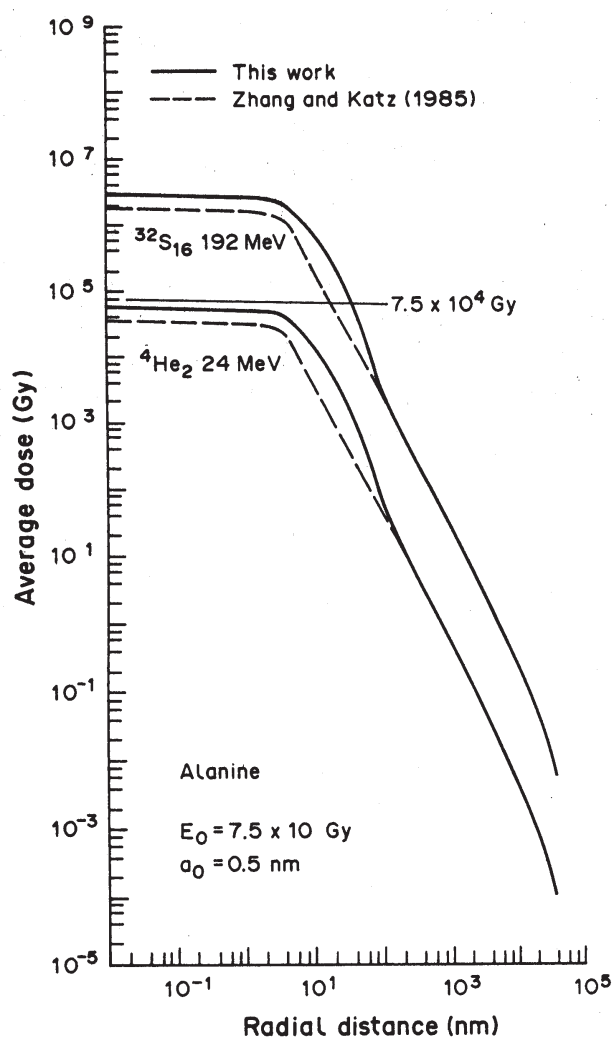

Figure 6. Radial distributions of dose averaged over a sensitive site of radius $a_{0}=0.5 \mathrm{~nm}$, calculated using the radial distribution of dose formula given by equation (2) (full lines) and that without the term $K(r)$ in equation (2) (broken lines; Zhang et al. 1985), for $S$ (upper curves) and He (lower curves) of energy $6 \mathrm{MeV} /$ a.m.u. The horizontal line at $E_{0}=7.5 \times 10^{4} \mathrm{~Gy}$ represents the detector saturation level.

and using equation (2) are compared. The pronounced effect of including the term $K(r)$ on the results of calculations of the response for the lighter He ion is explained qualitatively in Figure 6. Here, the corresponding radial distributions of dose averaged over the volume of the sensitive site $\left(a_{0}=0.5 \mathrm{~nm}\right)$, calculated using both forms, are shown for the He and $\mathrm{S}$ ions at their initial energies of $6 \mathrm{MeV} /$ a.m.u. The relative positions of the flat central parts of the radial distributions of average dose $E(t)$ [see equation (3)] and the saturation dose $\left(E_{0}=7.5 \times 10^{4}\right.$ Gy) determine the value of the calculated response. In the case of the sulphur ion, the flat portions of the $E(t)$ curves lie high above the saturation dose value, therefore the calculated relative effectiveness is much less than 1 and does not strongly depend on the differences in the shapes and positions of the two curves. The central parts of the $E(t)$ distributions corresponding to the helium ion both lie below $E_{0}$, therefore the value of the calculated response is strongly dependent on differences in the shapes of $E(t)$ in relation to the value of $E_{0}$.

The inability to fit a "physically" measured value $E_{0}$ $=1.05 \times 10^{5}$ Gy without modifying the maximum range $\tau$ of the radial distributon of dose $D(r)$ also raises in- teresting questions. A comparison between the radial dose distributions measured in gases and the radial distribution of dose formula [equation (2)] where $\tau$ is unchanged ("range factor" $=1$ ) or divided by 2 or by 5 ["range factors" of $1 / 2$ or $1 / 5$, respectively, equation (A. 10)] is shown in Figure 7. It appears that modifying the shape of the radial distribution of dose by dividing $\tau$ by a factor of up to 5 would still be compatible with measurements and results of Monte Carlo calculations (see also, Waligórski et al. (1987b)). We have tested the effect of modifying $\tau$ on the value of the radial integral of $D(r)$ [equation (12)]. For protons whose energies lie in the range from 1000 to $1 \mathrm{MeV}$, reducing the value of $\tau$ to a half of its value decreases the value of the integral by less than $7 \%(2,3,4$, and $6 \%$ at energies of 1000,100 , 10 , and $1 \mathrm{MeV}$, respectively), while reducing $\tau$ to $\tau / 5 \mathrm{de}-$ creases the value of this integral by up to $17 \%(5,7,10$, and $17 \%$, respectively). For a $0.25 \mathrm{MeV}$ proton, reducing $\tau$ to half its value decreases the integral value by $16 \%$; five-fold reduction decreases it by $40 \%$.

In all, the variant of track structure calculations, where the maximum range of the $\delta$-ray dose distribution formula $\tau$ is reduced by one-half, gives a reasonable fit to the experimental data set and reasonable, "physically correct," values of radiosensitivity parameters for alanine: a value of $E_{0}$ which is actually measured (Hansen and Olsen, 1985) and a value of $a_{0}=2 \mathrm{~nm}$ which corresponds to the value expected from target theory considerations (Dertinger and Jung, 1970; Katz and Hermann, 1976).

Analyzing the results of both variants of our calculations, we may conclude that our presently used formula (Zhang et al., 1985) describing the radial distribution of dose may overestimate the dose in the region over which the correction $K(r)$ has been introduced, perhaps by a factor of 2 , and may also overestimate its penetration range, by about the same factor, both factors perhaps being dependent on the ion's speed.

In view of these findings, it is likely that the importance of the constraint imposed by equation (12) to the development of more accurate formulations of the radial distribution of dose will have to be reconsidered.

It should be stressed at this point that our track structure theory is a purely phenomenological one, hence the correctness of any of its elements must ultimately be judged by the correctness with which model calculations can reproduce experimental measurements for as wide a range of detectors as possible. Other elements of the theory: the description of the effective charge, representing the medium of our detectors by water, application of simplified energy-range relationships for electrons, etc., will also contribute to its overall accuracy. It is gratifying, however, that our calculations, using a simple three-parameter representation of a $c$-hit detector and the present formulation of the radial distribution of dose (Waligórski et al., 1987b), can usually reproduce its 


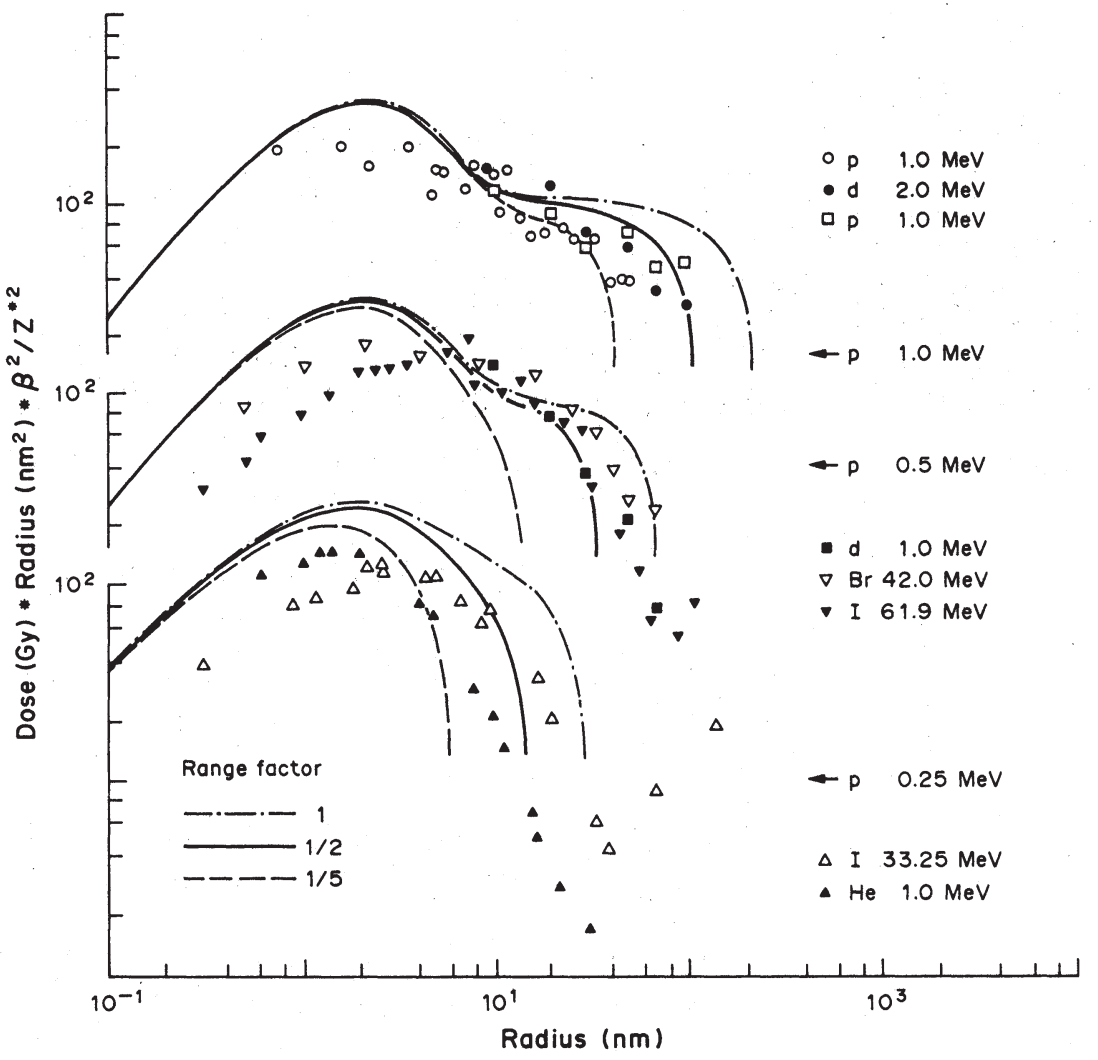

Figure 7. Measured radial distribution of dose, multiplied by the square of the radial distance and by $\beta^{2} / Z^{* 2}$, for ions of $1 \mathrm{MeV} /$ a.m.u. (uppermost group), $0.5 \mathrm{MeV} /$ a.m.u. (central group) and $0.25 \mathrm{MeV} /$ a.m.u. (lowest group). Full lines represent equation (2) where factors dividing the maximum $\delta$-ray range $\tau$ [equation (A. 10)] by 1, 2, and 5 were applied. Key to sources of data: 1 MeV

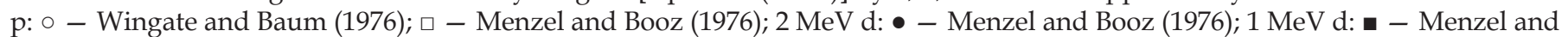
Booz (1976); $42 \mathrm{MeV}$ Br: $\nabla$ - Varma et al. (1980); $61.9 \mathrm{MeV}$ I: $\boldsymbol{\nabla}$ - Baum et al. (1974);1 MeV He: $\boldsymbol{\Delta}$ - Wingate and Baum (1976); 33.25 MeV I: $\Delta$ - Baum et al. (1974).

response after high-LET radiations to within $15 \%$ or better, as has already been shown also for enzyme and virus inactivation (Waligórski et al., 1987c) and the Fricke dosimeter (Katz et al., 1986).

Judging from the overall success of our average-dose model approach, one may propose that knowledge of the detailed spectrum of energy depositions in nanometer or micrometer subvolumes and its dependence on the separation of these volumes may be superfluous when interpreting experimental data at the present level of accuracy.

Within this perspective, detailed ab initio models of the interaction of the detector media and photons and electrons and better experimental and theoretical determinations of the radial distribution of $\delta$-rays around the path of a heavy ion transversing the detector media, rather than arbitrary specifications of "core" and "penumbra" ion track interaction regions, are more likely to contribute to further developments in heavy ion and neutron dosimetry using one-hit detectors.

\section{Conclusions}

A survey of experimental measurements of the relative effectiveness of alanine, a one-hit detector, after charged particle and neutron irradiations, and the subsequent track structure theory calculations, have yielded the following radiosensitivity parameters representing alanine: $E_{0}=1.05 \times 10^{5} \mathrm{~Gy}$, a value measured experimentally, and a value of $a_{0}=2 \mathrm{~nm}$. To achieve this fit, the formula describing the radial distribution of dose around the path of a heavy ion had to be modified. With no adjustment of the radial dose formula, a different set of parameters: $E_{0}=7.5 \times 10^{4}$ Gy and $a_{0}=0.5 \mathrm{~nm}$ could be fitted to experimental data. This may suggest that the presently used formula describing the radial distribution of $\delta$-ray dose around the path of a heavy ion should perhaps be modified by decreasing its range of radial penetration by about one half. Such a modification is compatible with other assessments of the shape of the radial distribution of dose.

We have shown that response of alanine after charged particle and neutron irradiations can be calculated theoretically and experimental results predicted roughly to within experimental accuracy (10-15\%), except for proton and $\alpha$-particle irradiations, where the calculation appears to underestimate the measured response. This could indicate that, in its central region, our formula somewhat overestimates the dose around the path of a heavy ion. In some cases, the use of "equivalent radia- 
tion" to estimate the response of alanine to beams of fast neutrons could be advantageous.

Thus, track structure calculations are able to support practical high-LET dosimetry using alanine.

While the overall results of this work should be considered a success of track structure theory, the alanine detector will continue serving as a test of this theory. Future measurements of the response of alanine after "light" ions (energetic protons and $\alpha$-particles) in tracksegment and stopping-particle configurations could provide us with particularly valuable means of testing the overall assumptions of track structure theory, and allow us to better determine the shape of the radial distribution of $\delta$-ray dose around the path of a heavy ion.

\section{Acknowledgments}

We wish to thank Dr. Dan Schlitt for his advice on software and hardware problems, and Mr. Gary Sinclair for his extensive help in developing computer codes and graphics. One of the authors (M.P.R.W.) has been supported by a fellowship from the International Atomic Energy Agency (Vienna) at the time this work was carried out. This work was supported by the United States Department of Energy.

\section{References}

Bach R. L. and Caswell R. S. (1968) Energy transfer to matter by neutrons. Radiat. Res. 35, 1-25.

Baum, J. W., Varma M. N., Wingate C. L., and Kuehner A. V. (1974) Nanometer dosimetry of heavy ion tracks. In Proc. Fourth Symp. on Microdosimetry (Eds. Booz J., Ebert H. G., Eickel R. and Waker A.). Commission of the European Communities, Luxembourg.

Bermann F. (1978) Adaptation of a free radial dosimeter to the intercomparison of fast neutron beams. IAEA Contract No. 1542/R2/RB, Final Report (unpublished).

Bradshaw W. W., Cadena D. G. Jr, Crawford G. W., and Spetzler H. A. W. (1962) The use of alanine as a solid dosimeter. Radiat. Res. 17, 11-21.

Butts J. J. and Katz R. (1967) Theory of RBE for heavy ion bombardment of dry enzymes and viruses. Radiat. Res. 30, 855-871.

Caswell R. S. and Coyne J. J. (1972) Interaction of neutrons and secondary charged particles with tissue: Secondary particle spectra. Radiat. Res. 52, 418-470.

Deffner U. and Regulla D. F. (1980) Influences of physical parameters on high-level amino acid dosimetry, Proc. 6th Int. Conf. on Solid State Dosimetry, Toulouse, 1-4 April, 1980. Nucl. Instrum. and Methods 175, 134-135.

Dertinger H. and Jung H. (1970) Molecular Radiation Biology. Springer Verlag, New York.

Ebert P. J., Hardy K. A., and Cadena D. G. (1965) Energy dependence of free radical production in alanine. Radiat. Res. 26, 178-197.

Hansen J. W. (1984) Experimental investigation of the suitability of the track structure theory in describing the relative effectiveness of high-LET irradiation of physical radiation detectors. Risø-R-407, Risø National Laboratory, Denmark.

Hansen J. W. and Olsen K. J. (1984) Experimental and calculated response of a radiochromic dye film dosimeter to high-LET radiations. Radiat. Res. 97, 1-15.
Hansen J. W. and Olsen K. J. (1985) Theoretical and experimental radiation effectiveness of the free radical dosimeter alanine to irradiation with heavy charged particles. Radiat. Res. 104, 15-27.

Hansen J. W., Olsen K. J., and Wille M. (1987) The alanine radiation detector for high and low LET dosimetry. Radiat. Project. Dosim. 19, 43-47.

Henriksen T. (1966) Production of free radicals in solid biological substances by heavy ions. Radiat. Res. 27, 676-693.

Katsumura Y., Tabata Y., Seguchi T., Morishita N., and Kojima T. (1986) Fast neutron irradiation effects-III. Sensitivity of alanine systems for fast neutron having an energy ofc. $1 \mathrm{MeV}$. Radiat. Phys. Chem. 28, 337-341.

Katz R. (1978) Track structure theory in radiobiology and in radiation detection. Nucl. Track Detection 2, 1-28.

Katz R. and Bermann F. (1976) Un dosimetre a large gamme, peu sensible a la qualite dur rayonnement: l'alanine. 8-eme Congres International de la Societe Francaise de Radioprotection, Saclay, 2326 March 1976.

Katz R., Sharma S. C., and Homayoonfar M. (1972a) The structure of particle tracks. In Topics in Radiation Dosimetry, (Ed. Attix, F.) Academic Press, New York.

Katz R., Sharma S. C., and Homayoonfar M. (1972b) Detection of energetic heavy ions. Nucl. Instrum. Methods 100, 13-32.

Katz R., Sinclair G. L., and Waligórski M. P. R. (1986) The Fricke dosimeter as a 1-hit detector. Nucl. Tracks Radial. Meas. 11, 301-307.

Menzel H. G. and Booz J. (1976) Measurement of radial energy deposition spectra for protons and deuterons in tissue equivalent gas. In Proc. Fifth Symposium on Microdosimetry (Eds Booz J., Ebert G. H. and Smith B. G. R.). Commission of The European Communities, Luxembourg.

Mueller A., Schambra P. E., and Pietsch E. (1964) Comparative ESR measurements of radical production in amino-acids by ${ }^{210}$ Po-alpha- and ${ }^{60} \mathrm{Co}$ gamma-radiation. Int. J. Radiat. Biol. 7, 587-599.

Regulla D. F. and Deffner U. (1982) Dosimetry by ESR spectroscopy of alanine. Int. J. Appl. Radiat. Isot. 33, 1101-1114.

Rotblat J. and Simmons J. A. (1963) Dose-response relationships in the yield of radiation-induced free radicals in amino-acids. Phys. Med. Biol. 7, 489-497.

Simmons J. A. (1987) Analysis of the dose-response relationships following the irradiation of amino-acids. Radiat. Res. 111, 374-377.

Simmons J. A. and Bewley D. K. (1976) The relative effectiveness of fast neutrons in creating stable free radicals. Radiat. Res. 65, 197-201.

Snipes W. and Horan P. K. (1967) Electron spin resonance studies of free radical turnover in gamma-irradiated single crystals of alanine. Radiat. Res. 30, 307-315.

Varma M. N., Baum J. W., and Kuehner A. V. (1980) Stopping power and radial dose distribution for $42 \mathrm{MeV}$ bromine ions. Phys. Med. Biol. 25, 651-656.

Waligórski M. P. R. (1987) Two Lectures on Track Structure, Report No. 137 I/PL, Institute of Nuclear Physics, Krakow, Poland.

Waligórski M., Katz R., Byrski E., Sarna T., Pasenkiewicz-Gierula M., and J. Knapczyk (1981) Mixed-field dosimetry in a cyclotron produced fast neutron radiotherapy beam with special emphasis on a newly developed alanine system. In Proc. 4th Symp. on Neutron Dosimetry, Munich-Neuherberg, 1-4 June 1981. Vol. 2, pp. 243-250.

Waligórski M. P. R., Hansen J. W., and Byrski E. (1987a) Appli- 
cation of the alanine detector to gamma-ray, $x$-ray and fast neutron dosimetry. Report No. 1373/PL, Institute of Nuclear Physics, Krakow, Poland, 1987. (Submitted to Nukleonika.)

Waligórski M. P. R., Hamm R. N., and Katz R. (1987b) The radial distribution of dose around the path of a heavy ion. Nucl. Tracks Radiat. Meas. 11, 309-319.

Waligórski M. R. P., Kirn Sun Loh, and Katz R. (1987c) Inactivation of dry enzymes and viruses by energetic heavy ions. $R a-$ diat. Phys. Chem. 30, 201-208.

Wingate C. L. and Baum J. W. (1976) Measured radial distributions of dose and LET for alpha and proton beams in hydrogen and tissue-equivalent gas. Radiat. Res. 65, 1-19.

Zhang Chunxiang, Dunn D. E., and Katz R. (1985) Radial distribution of dose and cross-sections for the inactivation of dry enzymes and viruses. Radiat. Protect. Dosim. 13, 215-218.

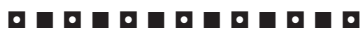

\section{Appendix}

\section{Radial Distribution of Dose}

In the present work, we apply a new set of formulae describing the radial distribution of dose around the path of a heavy ion, $D(r)$, elaborated elsewhere (Waligórski et al., 1987b), as summarized below.

Equation (2) in the text:

$$
D(r)=D_{1}(r)(1+K(r))
$$

where

$$
\begin{aligned}
\text { (a) for } r>B & =0.1 \mathrm{~nm}: \\
K(r) & =(A(r-B) / C) \exp (-(r-B) / C) \\
B & =0.1 \mathrm{~nm} \\
C & =1.15 \mathrm{~nm}+5 \mathrm{~nm} \times \beta
\end{aligned}
$$

and

$$
A \quad=8 \times \beta^{1 / 3} \text { for } \beta<0.03
$$

or

$$
\begin{array}{ll}
A & =19 \times \beta^{1 / 3} \text { for } \beta>0.03 \\
\text { (b) for } \quad r<B & =0.1 \mathrm{~nm} \text { : } \\
K(r) & =0
\end{array}
$$

and

$$
D_{1}(r)=\frac{N e^{4} Z^{* 2}}{\alpha m c^{2} \beta^{2} r} \frac{(1-(r+\theta) /(\tau+\theta))^{1 / \alpha}}{(r+\theta)}
$$

consists of two $r$-dependent terms, $K(r)$ and $D_{1}(r)$.

$D_{1}(r)$ represents the dose deposited in a coaxial cylindrical shell of thickness $\mathrm{d} r$ at a distance $r$ from the path of an ion of effective charge $Z^{*}$ moving with a relative velocity $\beta=v / c$ (c is the speed of light) through the detector medium containing $N$ electrons per $\mathrm{cm}^{3}, m$ is the mass of the electron. The Rutherford cross-section for $\delta$-ray production from atoms having ionization potential $I=10 \mathrm{eV}$, normal ejection and a power law range $(t)$-energy $(w)$ relationship for electrons, are assumed. The range-energy relationship is based on a two-component fit to the available experimental data concerning ranges of electrons in aluminum:

$$
t=k w^{\alpha}
$$

where

$$
k=6 \times 10^{-6} \mathrm{~g} \mathrm{~cm}^{-2} \mathrm{keV}^{-\alpha} .
$$

For

$$
w<1 \mathrm{keV}, \alpha=1.079 ; \text { for } w>1 \mathrm{keV}, \alpha=1.667 \text {. }
$$

$\theta$ is the "range" of an electron of energy $w=I$, viz.

$$
\theta=k \times(0.010 \mathrm{keV})^{1.079}=4.17 \times 10^{-8} \mathrm{~g} \mathrm{~cm}^{-2} .
$$

The kinematically limited maximum $\delta$-ray energy is:

$$
W=2 m c^{2} \beta^{2} /\left(1-\beta^{2}\right) \text {. }
$$

This translates to the maximum range of $\delta$-rays:

$$
\tau=k W^{\alpha}
$$

where the choice of $\alpha$ depends on the relative velocity $\beta$ of the ion. We calculate:

for

and for

$$
\beta<0.03, \alpha=1.079 \text {, }
$$

$$
\beta>0.03, \alpha=1.667 .
$$

In our calculations, where indicated, we have modified the value of $\tau$ by dividing it by factors 1 (no change), 2, and 5 . This, through expression (A.4), modifies the overall shape of the radial distribution of dose, as may be clearly seen in Figure 7. For energetic ions, only the outermost part of this distribution will be modified. Therefore the value of the integral of equation (12) in the main text will not change much with modifications of $\tau$ if the ion is energetic enough (above $1 \mathrm{MeV} /$ a.m.u.).

For water

$$
\begin{gathered}
\left(2 \pi N e^{4}\right) /\left(m c^{2}\right)=1.369 \times 10^{-7} \mathrm{erg} / \mathrm{cm} \\
=8.5 \mathrm{keV} \mathrm{mm}^{-1} .
\end{gathered}
$$

The effective charge number of an ion of atomic number $Z$ moving with relative speed $\beta$ is

$$
Z^{*}=Z\left(1-\exp \left(-125 \times \beta \times Z^{-2 / 3}\right)\right) \text {. }
$$

In the preceding formulae the expression $D_{1}(r)$ was calculated from the Rutherford formula, and includes only about one half of the total energy deposited by the ion. Provisionally, we think of it as the energy deposited by the $\delta$-rays. The contribution $K(r) \times D_{1}(r)$ is generated from a Monte Carlo calculation of the radial distribution of dose in liquid water, and provisionally is thought to represent the primary excitation energy contributed by the ion. In its complete form, when integrated radially [equation (12)], $D(r)$ is designed to reproduce the stopping power of a proton in liquid water to within $10 \%$, over a wide range of proton speeds. The contribution from $K(r)$ principally appears as a "hump" in a plot of the radial dose distribution at radial distances $1-10 \mathrm{~nm}$, and affects the shape of the central part of the radial distribution of dose averaged over the volume of the sensitive element, as indicated in Figure 6. 